\title{
Neoadjuvant chemoradiotherapy followed by laparoscopic distal gastrectomy with D2 lymph node dissection for a locally advanced distal gastric cancer
}

\section{Ziyu Li}

Beijing Cancer Hospital

Zining Liu ( $\nabla$ zining-liu96@hotmail.com )

Beijing Cancer Hospital https://orcid.org/0000-0002-4355-6899

\section{Yinkui Wang}

Beijing Cancer Hospital

\section{Fei Shan}

Beijing Cancer Hospital

\section{Shuangxi Li}

Beijing Cancer Hospital

Jiafu Ji

Beijing Cancer Hospital

\section{Case report}

Keywords: Gastric cancer, Neoadjuvant chemoradiotherapy, Laparoscopic, Distal gastrectomy

Posted Date: February 11th, 2020

DOI: https://doi.org/10.21203/rs.2.23113/v1

License: (c) (1) This work is licensed under a Creative Commons Attribution 4.0 International License.

Read Full License

Version of Record: A version of this preprint was published at World Journal of Clinical Cases on April 16th, 2021. See the published version at https://doi.org/10.12998/wjcc.v9.i11.2542. 


\section{Abstract}

Laparoscopic technique has been widely applied for early gastric cancer, with the advantages of minimal invasion and quick recovery. However, there is no report about the safety and short-term outcome of laparoscopic gastrectomy with D2 lymph node dissection for patients after neoadjuvant chemoradiotherapy. We presented the first case treated in this way. The patient was a 60-year-old man who was diagnosed with advanced distal gastric cancer. The neoadjuvant chemoradiotherapy was performed based on the regimen of GTV 50Gy/25f and CTV 45Gy/25f, as well as concurrent S-1 60mg Bid. Radiological examination determined that a partial response (PR) had been achieved by the initial therapy. Adverse events included only a myelosuppression limited to grade 2 . Then laparoscopic distal gastrectomy with D2 lymph node dissection was undertook successfully for him. The patient recovered smoothly with no postoperative complications. The postoperative pathological stage was ypT3NOM0, with necrosis rate $>90 \%$. He was still in good condition after five years follow-up.

\section{Introduction}

Since the first case of laparoscopic distal gastrectomy (LDG) was reported by Kitano in 1994 [1], laparoscopic technique has been widely applied for early gastric cancer, with the advantages of minimal invasion and quick recovery[2-4]. By now laparoscopic gastric surgery has become a preferred option for early gastric cancer. The promising outcome has promoted the application of laparoscopy in advanced gastric cancer. Although it has not reach an agreement on whether laparoscopic gastrectomy was suitable for locally advanced gastric cancer, the safety and feasibility of laparoscopic gastrectomy has been explored by a few clinical trials[5-9]. And inspiring results have been achieved by them, proving that laparoscopic gastrectomy for locally advanced gastric cancer is not inferior to that of open surgery.

Although surgery is the only curative option for gastric cancer, the mortality of patients after radical surgery remains high because of a significant number of local regional or systemic recurrences[10]. Chemoradiotherapy have been recommended as a supplement to radical surgery by the National Comprehensive Cancer Network[11]. Sometimes post-operative adjuvant chemoradiotherapy administration was restricted in patients due to surgical complications. However, the application of chemoradiotherapy before surgery can avoid the shortcoming. Meanwhile, neoadjuvant chemoradiotherapy has the advantages in avoiding unnecessary surgery by shrinking tumor size and facilitating high rate of R0 resection, with acceptable rates of acute and late toxicity[12-14].

To our knowledge, this is the first report about the safety and short-term oncological outcome of neoadjuvant chemoradiotherapy followed by laparoscopic D2 gastrectomy, to date.

\section{Case Report}

\section{Pretreatment diagnosis}


A 60-year-old man was presented with abdominal distension and weight loss of over $10 \mathrm{~kg}$ for about 6 months' duration. He was an otherwise healthy man with no remarkable medical history. On admission, the physical examination showed no signs of abdominal abnormalities. His tumor markers were normal expect carcinoembryonic antigen (CEA). The serum CEA level was $3.44 \mathrm{ng} / \mathrm{ml}$, slightly higher than normal. His other laboratory values were within the normal limits.

The endoscopy was undertaken on May 23rd of 2013, revealing an ulcerative lesion adjacent to the gastric antrum, with dike-like bulge around the edge. Pylorus was invaded by the lesion and became incomplete obstruction. (Fig. 1) The pathology of endoscopic biopsy confirmed poorly differentiated adenocarcinoma, with the Lauren type of intestine- type. Enhanced abdominal computed tomography (CT) (2013-5-29) indicated that the wall of the antrum was thickened with significant enhancement. The maximum thickness was $21 \mathrm{~mm}$ (Fig. 2). The surface of serosa was fuzzy, but the border near the pancreas was still clear. Multiple enlarged lymph nodes were found in the greater and lesser gastric curvature, the diameter of the largest one was $7 \mathrm{~mm}$. No distant metastasis was detected by pelvis or chest CT. The patient was diagnosed as gastric antrum carcinoma, Borrmann type III, with lymph nodes metastases.

\section{Neoadjuvant chemoradiotherapy and evaluation}

Based on the previous imaging detection, clinical stage was confirmed as cT4aNxMO III. According to the NCCN guideline for gastric cancer, neoadjuvant chemoradiotherapy followed by D2 gastrectomy was recommended by multidisciplinary team (MDT). The neoadjuvant chemoradiotherapy was given to a total dose of GTV 50 Gy, CTV 45 Gy, in 25 fractions, 5 times a week, with concurrent S-1 $60 \mathrm{mg}$ Bid, from $2013 / 6 / 17$ to $2013 / 7 / 16$. The neoadjuvant chemoradiotherapy was well tolerated with no severe adverse events. The adverse events were as follows: bone marrow suppression $\mathrm{II}^{\circ}$, digestive tract reaction $\mathrm{I}^{\circ}$, fatigue $l^{\circ}$. For incomplete obstruction, enteral nutrition was recommended for him. So the nutrition status of the patient was improved gradually during the neoadjuvant chemoradiotherapy.

Enhanced abdominal CT was performed to evaluate the response of neoadjuvant chemoradiotherapy at the time rightly (2013/7/12) and six weeks (2013/9/2) after neoadjuvant chemoradiotherapy, respectively (Fig. 3). Based on the imaging evaluation after preoperative chemoradiotherapy, a promising partial response (PR) was considered by MDT. Endoscopic ultrasound (EUS) was also applied to evaluate the response of neoadjuvant chemoradiotherapy, (2013/9/9) (Fig. 4) indicating the ulcerative lesion adjacent to the gastric antrum with pyloric stenosis. The lesion was reflected as hypoechoic signal under EUS, invading the whole layer, partly to the serosa. The maximum thickness was $1.09 \mathrm{~cm}$. A hypoechoic nodule beside the antrum, measuring about $2.05^{\star} 1.3 \mathrm{~cm}$ in size, was also detected. So it was restaged as uT4aN1M0 after neoadjuvant chemoradiotherapy.

\section{Surgery and postoperative recovery}


Open distal gastrectomy with D2 lymph node dissection was planned for the patient. However, the patient was so eager to receive minimally invasive surgery that he refused the open surgery. In our experience that neoadjuvant chemotherapy did not increase the complications of radical gastrectomy[15]. Considering that our center was experienced in laparoscopic D2 gastrectomy for locally advanced gastric cancer, we decided to undertake the laparoscopic D2 gastrectomy for the patient. The patient received laparoscopy-assisted distal gastrectomy with D2 lymph node dissection and Billroth II anastomosis successfully on $2013 / 10 / 10$. The whole procedure of surgery was recorded by video. The operation lasted for 240 minutes. The blood loss was $100 \mathrm{ml}$. The first flatus time was three days after the operation. Upper gastrointestinal radiography was performed seven days after the operation, indicating that gastric peristalsis was well with no anastomosis leakage. Then the patient was allowed to intake liquid diet. Postoperative hospital-stay was 12 days with no postoperative complications.

\section{Postoperative histology}

Postoperative pathological evaluation was completed seven days after surgery. Small groups of cancer cells were reported to be detected outside of the muscularis propria, accompanied by the formation of extracellular mucus pools, and surrounded by fibrosis and chronic inflammatory cells (Fig. 5). All of the above were in accord with the response to chemoradiotherapy, and the necrosis rate was $>90 \%$. No vascular embolus or perineuronal invasion was detected. No metastases were found in lymph nodes $(0 / 31$. Fibrosis was detected in some lymph nodes, considered as change post neoadjuvant chemoradiotherapy). No cancer cell was detected in the gastric stump or the duodenal stump. Therefore, the pathological stage was ypT3NOMO.

\section{Postoperative follow-up}

One month after the operation, the patient was back to the clinic for follow-up. The enhanced abdominal $\mathrm{CT}$ and all the laboratory values showed that the patient was generally in good condition. The postoperative adjuvant chemotherapy was conducted for him. Five years after the surgery, the patient was still in good condition, with no recurrence or metastasis.

\section{Discussion}

The number of lymph node dissection is regarded as the most objective indicator to evaluate the quality of D2 operation for advanced gastric cancer patients. Zhao $\mathrm{Y}$ et al [16] once reported a retrospective study of 659 patients who were treated by laparoscopic or open gastrectomy. They found that the average number of lymph node dissection in the laparoscopic gastrectomy and open gastrectomy group were 33.2 and 32.8, respectively. There was no significant difference between two groups. Meanwhile, the average time for the laparoscopic gastrectomy and open gastrectomy procedures did not differ significantly ( $211 \pm 56$ vs $204 \pm 41 \mathrm{~min}$ ), but bleeding during the operation in the laparoscopic group were significantly less than that in the open group ( $128 \pm 85 \mathrm{vs} 301 \pm 156 \mathrm{ml}, \mathrm{p}<0.001)$. Similar results were published by Pugliese et al [17] and Hwang et al [18]. The number of lymph node dissection was 31 in this case, which was comparable to the average number of lymph node dissection by laparoscopic or 
open gastrectomy. The blood loss was $100 \mathrm{ml}$, similar to that of laparoscopic gastrectomy for advanced gastric cancer. But the operation time was 240 minutes in our case, higher than that of previous reports. Based on our experience, as the effects of chemoradiotherapy, the severe fibrosis around stomach made the surgery more difficult and delicate. That was the reason why the operation time of the patient treated by neoadjuvant chemoradiotherapy was longer than those who did not. However, with the amplification effect of laparoscopy, laparoscopic gastrectomy was safe and feasible for advanced gastric patients who was treated by neoadjuvant chemoradiotherapy in experienced department. Besides that, it would not increase the postoperative complications.

An issue that needs to be addressed is that our case was attempted by a gastrointestinal expert familiar with laparoscopic distal gastrectomy for locally advanced gastric cancer, and intensive care unit backup was arranged preoperatively.

Although the patient in our department recovered smoothly with no postoperative complications, whether neoadjuvant chemoradiotherapy followed by laparoscopic gastrectomy will increase the postoperative complications or not are still under controversies. And the most frequent complications for gastric cancer patients after neoadjuvant chemoradiotherapy were reported as nonspecific surgical complications[19].

\section{Conclusion}

Neoadjuvant chemoradiotherapy followed by laparoscopic distal gastrectomy with D2 lymph node dissection was safe and effective for present patient. However, whether laparoscopic gastrectomy can be recommended as an initial treatment for advanced distal gastric cancer after neoadjuvant chemoradiotherapy, well-designed prospective trials are still needed to proof.

\section{Declarations}

\section{Abbreviation}

GTV: Gross tumor response; CTV: Clinical tumor response; PR: Partial response; LDG: Laparoscopic distal gastrectomy; CEA: Carcinoembryonic antigen; CT: Computed tomography; NCCN: National Comprehensive Cancer Network; MDT: Multidisciplinary team; EUS: Endoscopic ultrasound

\section{Conflict of interest:}

The author declares that he has no conflict of interest.

\section{Human rights statement and informed consent}

Informed consent was obtained from the patient before neoadjuvant chemoradiotherapy.

\section{Acknowledgement:}


This work was funded by Beijing Municipal Science and Technology Commission $₫ Z 151100004015070$, D141100000414002】

\section{Ethical Approval and Consent to participate}

This study is approved by institutional Ethics committee. Informed consent was obtained from the patient for the publication of this case report and any accompanying imaging and pathological data.

\section{Consent for publication}

Informed consent was obtained from the patient before neoadjuvant chemoradiotherapy.

\section{Availability of supporting data}

The datasets used and analyzed during the current study are available from the corresponding author on reasonable request.

\section{Competing interests}

The author declares that he has no conflict of interest.

\section{Funding}

This work was funded by Beijing Municipal Science and Technology Commission $₫ Z 151100004015070$, D141100000414002】

\section{Authors' contributions}

Z.Y.L, J.F.J and Z.N.L determined the preoperative regimen and performed the operation. The postoperative follow-up was done by Y.K.W, S.X.L and F.S. Z.N.L and Y.K.W collected patient data, performed image processing and composed manuscript. Z.Y.L revised and provided recommendation for the manuscript. All authors read and approved the final manuscript.

\section{Acknowledgements}

We acknowledge our patient and his family for providing informed consentfor this case report.

\section{Authors' information}

1. Key laboratory of Carcinogenesis and Translational Research (Ministry of Education), Department of Gastrointestinal Surgery, Peking University Cancer Hospital \& Institute, Beijing 100142, China

\section{References}


1. Kitano S, Iso Y, Moriyama M, Sugimachi K: Laparoscopy-assisted Billroth I gastrectomy. Surgical laparoscopy \& endoscopy 1994, 4(2):146-148.

2. Kitano S, Yasuda K, Shiraishi N: Laparoscopic surgical resection for early gastric cancer. European journal of gastroenterology \& hepatology 2006, 18(8):855-861.

3. Mochiki E, Kamiyama Y, Aihara R, Nakabayashi T, Asao T, Kuwano H: Laparoscopic assisted distal gastrectomy for early gastric cancer: Five years' experience. Surgery 2005, 137(3):317-322.

4. Tanimura S, Higashino M, Fukunaga Y, Kishida S, Ogata A, Fujiwara Y, Osugi H: Respiratory function after laparoscopic distal gastrectomy-an index of minimally invasive surgery. World J Surg 2006, 30(7):1211-1215.

5. Azagra JS, Ibanez-Aguirre JF, Goergen M, Ceuterick M, Bordas-Rivas JM, Almendral-Lopez ML, Moreno-Elola A, Takieddine M, Guerin E: Long-term results of laparoscopic extended surgery in advanced gastric cancer: a series of 101 patients. Hepatogastroenterology 2006, 53(68):304-308.

6. Kim MC, Kim HH, Jung GJ: Surgical outcome of laparoscopy-assisted gastrectomy with extraperigastric lymph node dissection for gastric cancer. European journal of surgical oncology : the journal of the European Society of Surgical Oncology and the British Association of Surgical Oncology 2005, 31(4):401-405.

7. Dulucq JL, Wintringer P, Perissat J, Mahajna A: Completely laparoscopic total and partial gastrectomy for benign and malignant diseases: a single institute's prospective analysis. Journal of the American College of Surgeons 2005, 200(2):191-197.

8. Uyama I, Sugioka A, Sakurai Y, Komori Y, Hanai T, Matsui H, Fujita J, Nakamura Y, Ochiai M, Hasumi A: Hand-assisted laparoscopic function- preserving and radical gastrectomies for advanced-stage proximal gastric cancer. Journal of the American College of Surgeons 2004, 199(3):508-515.

9. Kim MC, Kim KH, Kim HH, Jung GJ: Comparison of laparoscopy-assisted by conventional open distal gastrectomy and extraperigastric lymph node dissection in early gastric cancer. J Surg Onco/2005, 91(1):90-94.

10. Gunderson LL: Gastric cancer-patterns of relapse after surgical resection. Seminars in radiation oncology 2002, 12(2):150-161.

11. NCCN Guidelines Version 1.2015 Staging Gastric Cancer. 2015.

12. Lowy AM, Feig BW, Janjan N, Rich TA, Pisters PW, Ajani JA, Mansfield PF: A pilot study of preoperative chemoradiotherapy for resectable gastric cancer. Ann Surg Oncol 2001, 8(6):519-524.

13. Ajani JA, Winter K, Okawara GS, Donohue JH, Pisters PW, Crane CH, Greskovich JF, Anne PR, Bradley JD, Willett $C$ et al: Phase II trial of preoperative chemoradiation in patients with localized gastric adenocarcinoma (RTOG 9904): quality of combined modality therapy and pathologic response. Journal of clinical oncology : official journal of the American Society of Clinical Oncology 2006, 24(24):3953-3958.

14. Ajani JA, Mansfield PF, Janjan N, Morris J, Pisters PW, Lynch PM, Feig B, Myerson R, Nivers R, Cohen DS et al: Multi-institutional trial of preoperative chemoradiotherapy in patients with potentially 
resectable gastric carcinoma. Journal of clinical oncology : official journal of the American Society of Clinical Oncology 2004, 22(14):2774-2780.

15. Li ZY, Shan F, Zhang LH, Bu ZD, Wu AW, Wu XJ, Zong XL, Wu Q, Ren H, Ji JF: Complications after radical gastrectomy following FOLFOX7 neoadjuvant chemotherapy for gastric cancer. World J Surg Oncol 2011, 9:110.

16. Zhao Y, Yu P, Hao Y, Qian F, Tang B, Shi Y, Luo H, Zhang Y: Comparison of outcomes for laparoscopically assisted and open radical distal gastrectomy with lymphadenectomy for advanced gastric cancer. Surgical endoscopy 2011, 25(9):2960-2966.

17. Pugliese R, Maggioni D, Sansonna F, Scandroglio I, Ferrari GC, Di Lernia S, Costanzi A, Pauna J, de Martini P: Total and subtotal laparoscopic gastrectomy for adenocarcinoma. Surgical endoscopy 2007, 21(1):21-27.

18. Hwang SI, Kim HO, Yoo CH, Shin JH, Son BH: Laparoscopic-assisted distal gastrectomy versus open distal gastrectomy for advanced gastric cancer. Surgical endoscopy 2009, 23(6):1252-1258.

19. Valenti V, Hernandez-Lizoain JL, Beorlegui MC, Diaz-Gozalez JA, Regueira FM, Rodriguez JJ, Viudez A, Sola I, Cienfuegos JA: Morbidity, mortality, and pathological response in patients with gastric cancer preoperatively treated with chemotherapy or chemoradiotherapy. J Surg Oncol 2011, 104(2):124-129.

\section{Figures}

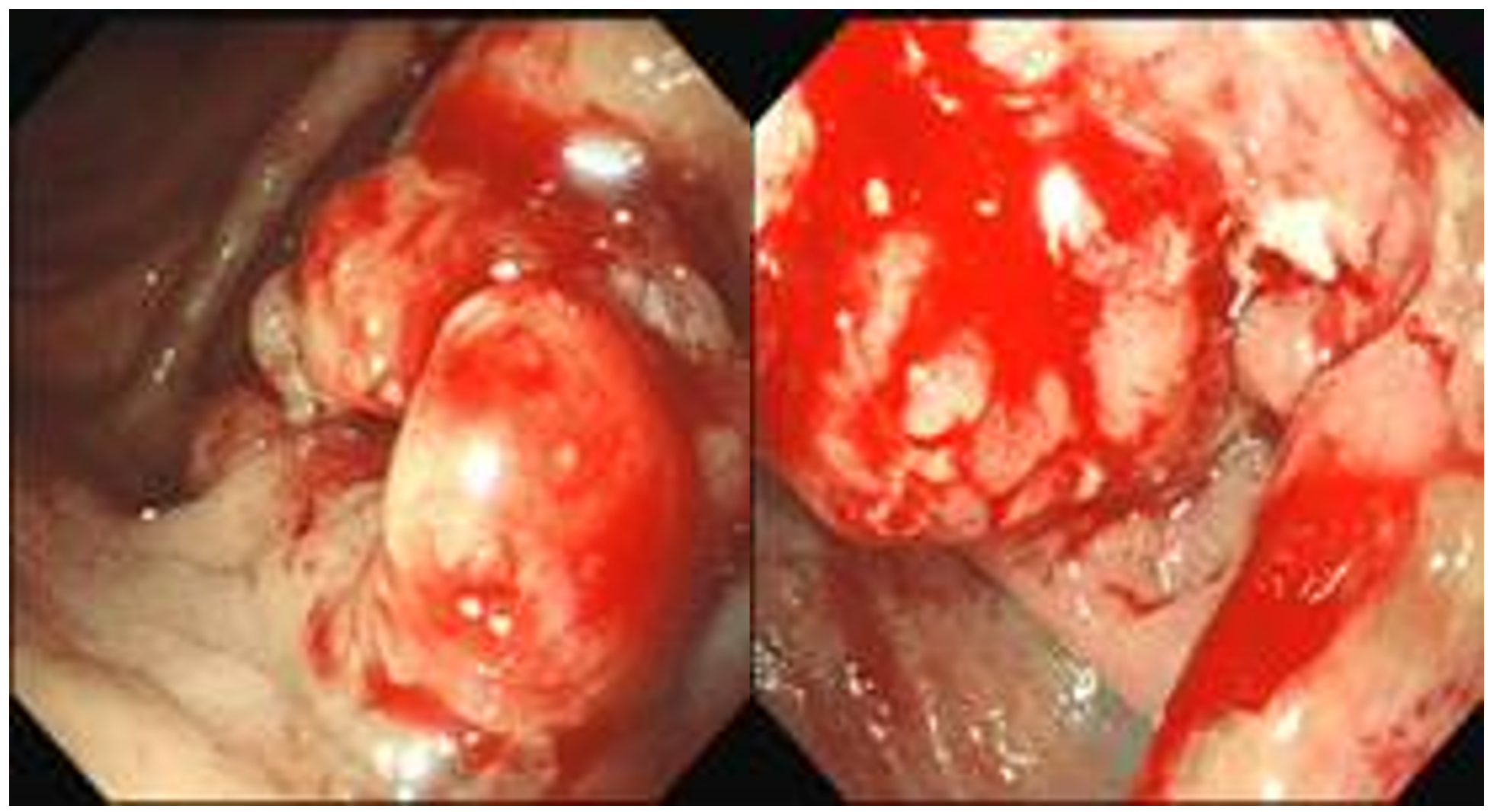

Figure 1 
Gastrointestinal fiberscope before chemoradiotherapy showed an ulcerative lesion located surrounding the gastric antrum with ulceration causing stenosis.
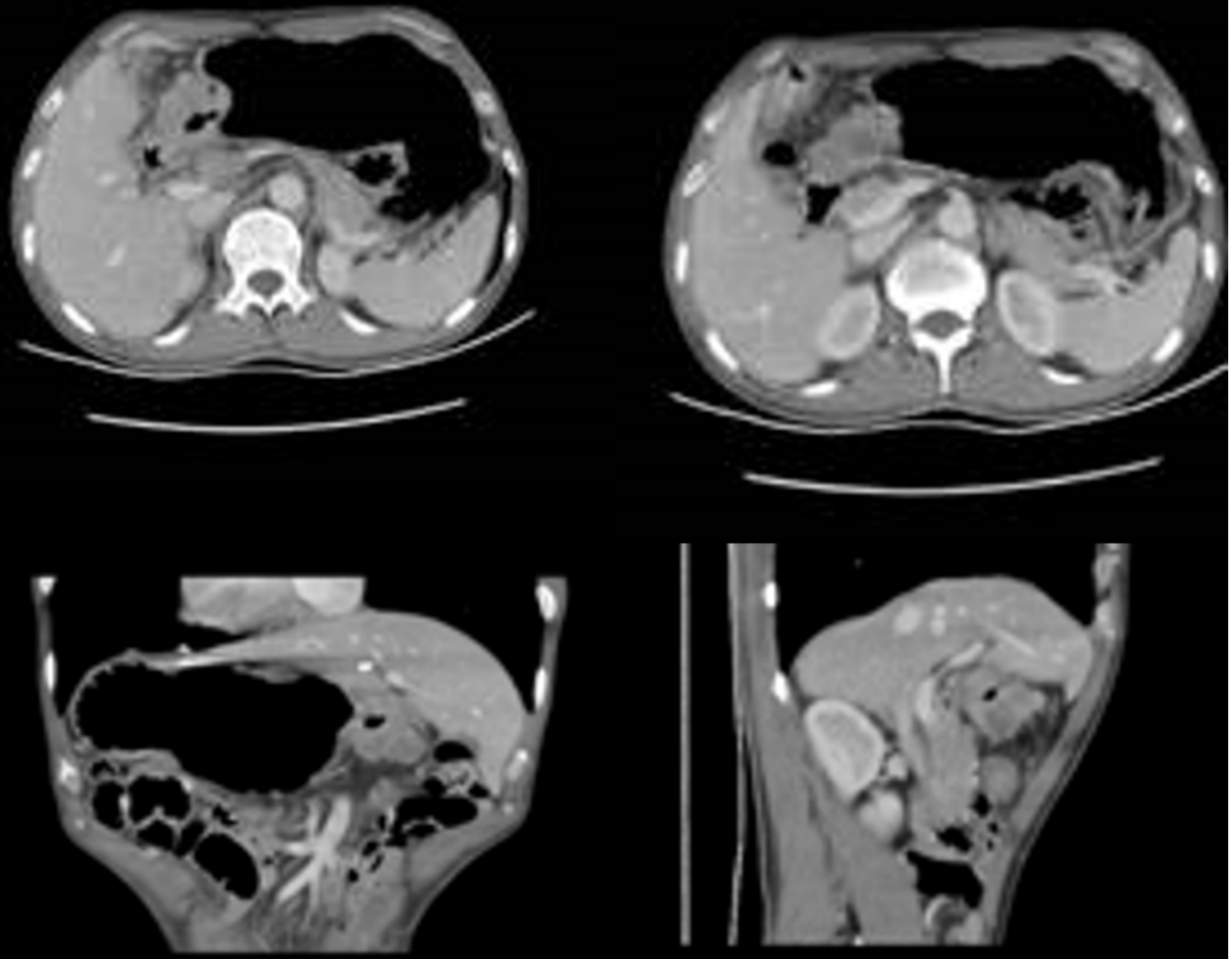

\section{Figure 2}

Enhanced abdominal CT indicated that the wall of the antrum was thickened with significant enhancement. The surface of serosa was fuzzy, but the border near the pancreas was still clear. Multiple enlarged lymph nodes were found in the greater and less gastric curvature. 

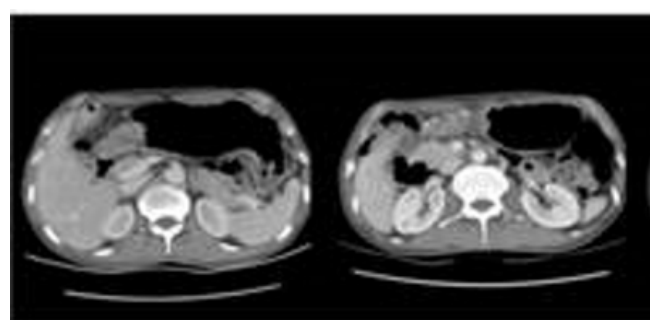

$2013-5 \cdot 29$

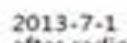

after radio.

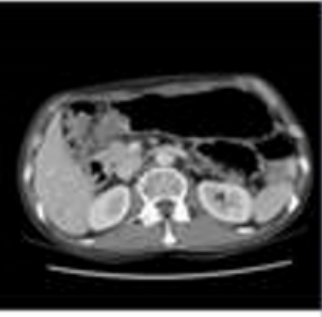

2013-9-2 6 w after radio.

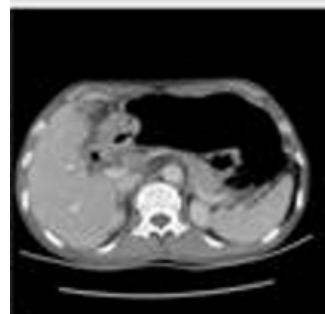

2013-5-29 betore radio.

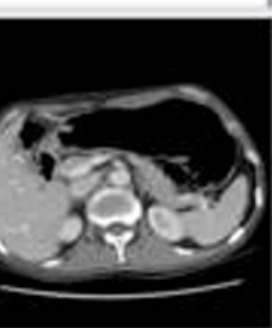

$2013 \cdot 9-2$

2013-7-1.

$6 \mathrm{w}$ after radio.
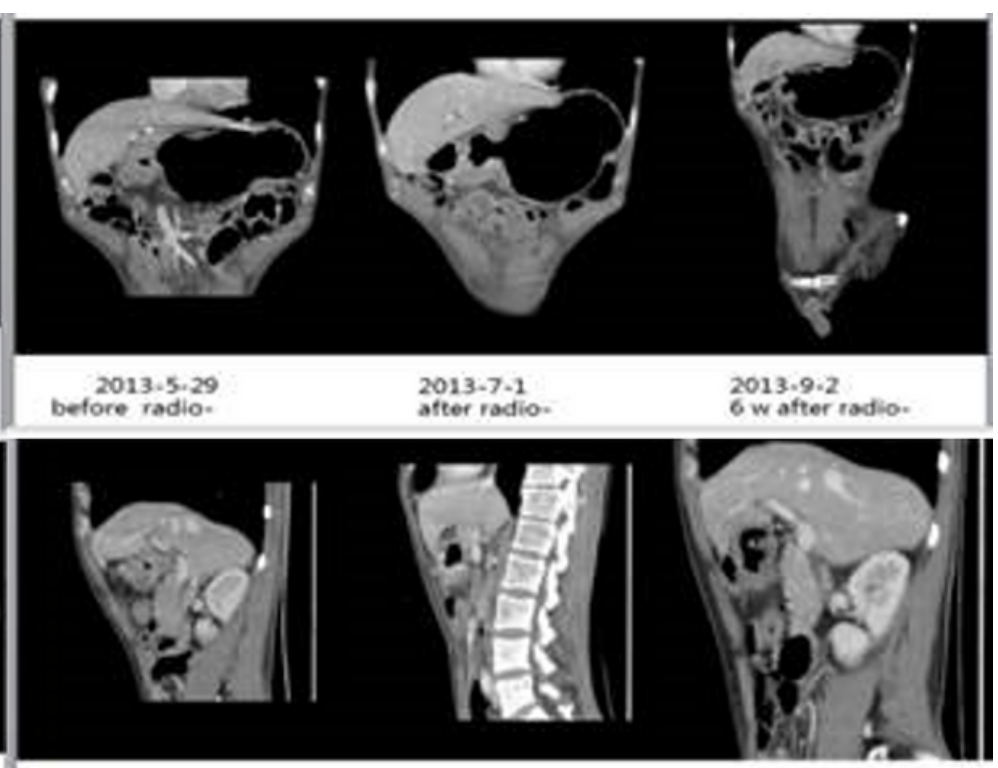

2013-5-29 betore radio.
$2013-7-1$
after radio-

$2013-9 \cdot 2$ 6 watter radio-

\section{Figure 3}

Enhanced abdominal CT performed at the time rightly and six weeks after neoadjuvant chemoradiotherapy respectively to evaluate the response to neoadjuvant chemoradiotherapy. At the first time, the maximum thickness changed from $21 \mathrm{~mm}$ to $12 \mathrm{~mm}$ (shrinking $42.9 \%$ ) with lymph nodes in the great curvature shrinking so obviously that they couldn't be clearly detected in the CT, but the surface of serosa was still fuzzy. The CT six weeks after neoadjuvant chemotherapy indicated the changing from $12 \mathrm{~mm}$ to $10 \mathrm{~mm}$ (shrinking $16.7 \%$ ) with no obviously enlarged lymph nodes around the stomach, retroperitoneal or pelvic. 


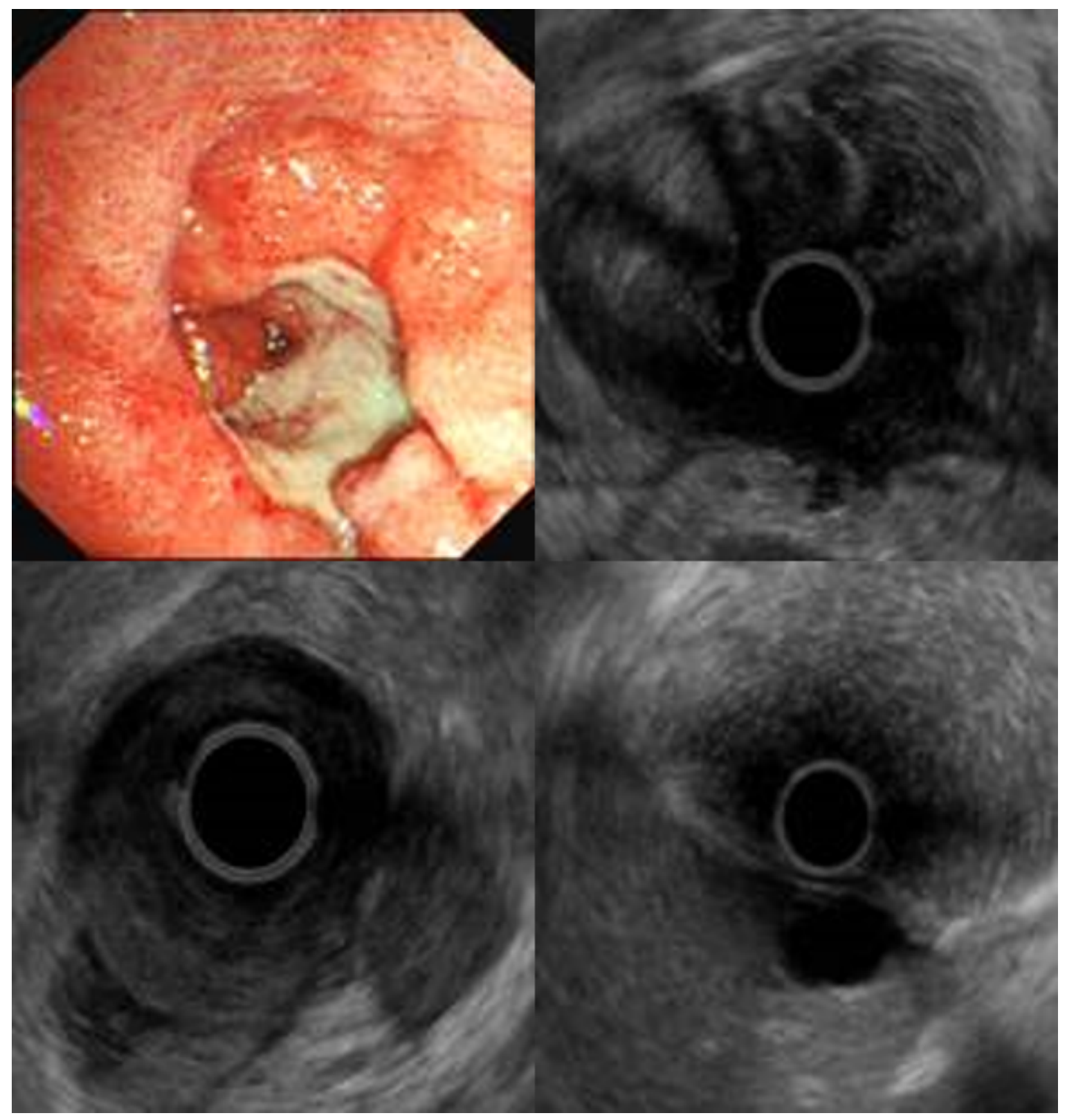

\section{Figure 4}

EUS was also applied to evaluate the response to neoadjuvant chemoradiotherapy, indicating the ulcerative lesion located surrounding the gastric antrum with pyloric stenosis. 


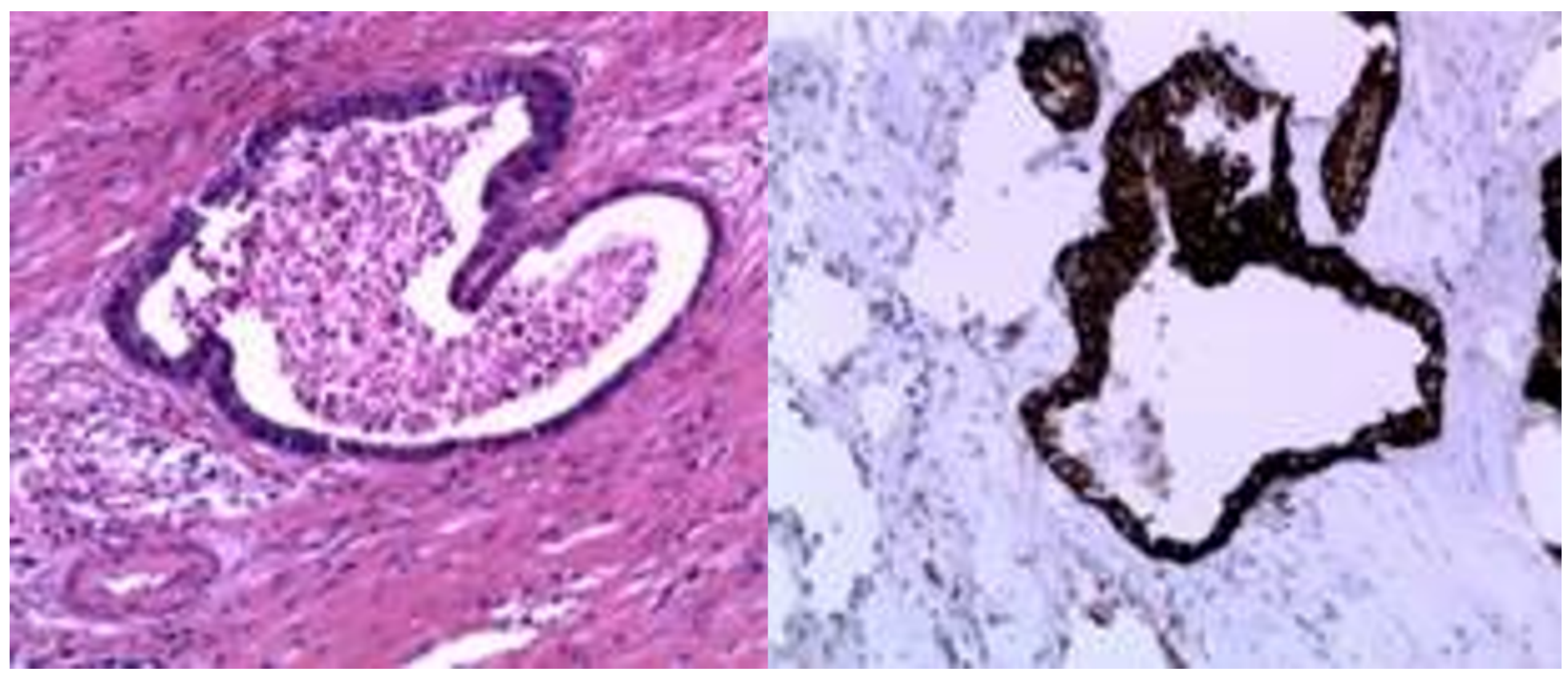

\section{Figure 5}

Postoperative pathological evaluation showed that the pathological stage after neoadjuvant chemoradiotherapy was T3NOMO, with necrosis rate $>90 \%$. 